\title{
Radio and microwave frequency signal generation and processing based on photonics using integrated Kerr optical micro-combs
}

david moss ( $\square$ dmoss@swin.edu.au )

Swinburne University of Technology https://orcid.org/0000-0001-5195-1744

\section{Research Article}

Keywords: Signal generator, Kerr micro-comb, RF signal processing

Posted Date: February 10th, 2022

DOI: https://doi.org/10.21203/rs.3.rs-1344379/v1

License: (c) (i) This work is licensed under a Creative Commons Attribution 4.0 International License.

Read Full License 


\section{Abstract}

We demonstrate a radio frequency (RF) phase-encoded signal generator as well as a user-defined RF arbitrary waveform generator (AWG) based on a soliton crystal micro-comb generated by an integrated MRR with a free spectral range of $\sim 49 \mathrm{GHz}$. Owing to the soliton crystal's robust and stable generation as well as the high intrinsic efficiency, RF phase-encoded signal generators and AWGs with simple operation and fast reconfiguration are realized. The soliton crystal micro-comb provides 60 wavelengths for RF phase-encoded signal generators, achieving a phase encoding speed of $5.95 \mathrm{~Gb} / \mathrm{s}$ and a high pulse compression ratio of 29.6. Over 80 wavelengths are employed for the AWGs, achieving tunable square waveforms with a duty cycle ratio ranging from $10-90 \%$, sawtooth waveforms with tunable slope ratios from 0.2 to 1 , and symmetric concave quadratic chirp waveforms. Our system has great potential to achieve RF and microwave photonic signal generation and processing with low cost and footprint.

\section{Introduction}

Phase-encoded signal generators are extensively exploited in low-probability-of-intercept radar (LPIR), since phase-encoded radio frequency (RF) signals feature low power density and employ random codes to avoid being cracked $[1,2]$. Arbitrary waveform generators (AWGs) are another significant category of signal sources with wider applications, such as radar, measurements, and wireless communications [35]. RF signals with broad bandwidth have great potential in these applications, for example achieving high-resolution target recognition in LPIR systems [6]. However, most of the existing approaches for RF phase encoded signal generators and AWGs are based on electronic technologies, with large footprint, high cost, and limitations in bandwidth and speed $[7,8]$.

Photonic techniques have significant potential to overcome limitations of their electronic counterparts benefiting from their intrinsically broad bandwidth, immunity from electro-magnetic interference, and low propagation loss. Extensive approaches have been developed to achieve photonic-assisted RF phase encoding, such as methods based on polarization modulators [9, 10], dual parallel modulators [11, 12], and Sagnac loops $[13,14]$. However, for these methods, there is a need for complicated modulation schemes, resulting in increased complexity and instability, and also a need for both high-frequency RF signal generators and AWGs, leading to increased cost, size, and power consumption. There are also limitations for approaches of achieving photonic-assisted RF arbitrary waveform generation. For spatialto-time and wavelength-to-time mapping methods [8,15-17], the synthesized waveforms are usually single-shot pulses. For Fourier synthesis method [18], the synthesized signal bandwidth is subject to the resolution of line-by-line spectral shaping.

An alternative method for photonic-assisted RF signal processing is based on transversal filter system, which offers high reconfigurability and accuracy attributing to the parallel scheme where each channel can be controlled independently. However, discrete laser arrays or electro-optical comb sources are employed to establish multiple wavelength channels. RF phase encoding based on transversal filter system has been proposed [19], where the need for complicated modulation schemes was reduced, but 
discrete laser arrays were used and high-order differentiation was implemented, which increased the complexity and cost of the system.

Integrated optical Kerr frequency combs, or 'micro-combs', have attracted significant interest for RF photonic systems as multi-wavelength sources. Micro-combs arise from optical parametric oscillation in ultra-high-Q monolithic micro-ring resonators (MRRs) and offer plenty of advantages compared with conventional multi-wavelength sources, such as larger wavelength number and greatly reduced system complexity and footprint. Extensive RF photonic applications have been demonstrated based on microcombs [20], such as transversal filters [21, 22], temporal signal processors [23-27], frequency synthesizers [28], true time delay lines [29], and channelizers [30,31] and many other applications [3243].

In this paper, we demonstrate an RF phase-encoded signal generator and a user-defined RF AWG based on a soliton crystal micro-comb generated by an integrated MRR with a free spectral range (FSR) of $\sim 49$ $\mathrm{GHz}[32,33]$. The soliton crystal micro-comb stably forms through the background wave generated by a mode crossing, and features over thirty times higher intra-cavity power than traditional dissipative Kerr solitons (DKS) [20]. The input RF signal is multicast onto the flatten micro-comb lines and progressively delayed via dispersion. For phase-encoded signal generation, 60 wavelengths are used and phase flipping is realized via differential photodetection. A phase encoding speed of $5.95 \mathrm{~Gb} / \mathrm{s}$ and a high pulse compression ratio of 29.6 are achieved. For arbitrary waveform generation, to enhance the speed and flexibility of the system, 81 wavelengths are used. Power of comb lines are tailored according to the designed weights. Tunable square waveforms with a duty cycle ratio ranging from $10-90 \%$, sawtooth waveforms with tunable slope ratios from 0.2 to 1 , and symmetric concave quadratic chirp waveforms with an instantaneous frequency reaching down to the sub- $\mathrm{GHz}$ range are achieved. These results verify the potential of our approaches as being an effective way to achieve RF phase-encoded signal generators and RF AWGs with low cost and footprint.

\section{Theory}

Figure 1(a) illustrate the operation principle of the photonic RF phase-encoded signal generator and AWG. First, an RF Gaussian pulse $f(t)$ with a duration of $\Delta t$ was generated. A soliton crystal micro-comb source, whose optical spectrum is shown in Figure 1(b), was generated by a Hydex MRR. Then a discrete convolution operation between the RF pulse and flattened micro-comb spectrum (denoted by a discrete signal $g[n]$ with length $N$ and binary values of 1 or -1 ) was performed with a delay step of $\Delta t$, and can be described as:

$$
(f * g)[n]=\sum_{i=1}^{N} f[n-i \cdot \Delta t] \cdot g[i]
$$

The discrete convolution operation between the RF pulse $f(t)$ and the flattened micro-comb spectrum was achieved using the experimental setup in Figure 1(c). The RF pulse was first broadcast onto the 
wavelength channels (comb lines flattened by a WaveShaper) to yield replicas, which were then delayed with a progressive step that matched with the pulse duration $\Delta t$.

For phase-encoded signal generation, by flipping the phase of the different delayed replicas via differential photodetection, according to designed phase codes $g[n]$, a phase-encoded sequence could be assembled in the time domain. The total number of RF pulse replicas $N$ is equal to the number of wavelength channels, which is 60 here-15 times that of previous work [19] - thus the total time length of the phase-encoded sequence is $T=N \cdot \Delta t$. Here, the basic temporal elements in the phase-encoded sequence are termed "RF segments", which are single-cycle or multi-cycle sine waves assembled from the input RF pulse. The center frequency of the phase-encoded sequence is determined by the frequency of the assembled sine waves, and thus is equivalently given by $1 /(2 \Delta t)$. Assuming each RF segment constitutes $m$ pulses, then the length of the phase-encoded sequence would be $N / m$, together with an equivalent phase encoding speed of $1 /(m \Delta t)$. We note that the generated phase-encoded sequence has a bandwidth similar to the input RF pulse, which is subject to the Nyquist bandwidth that is half of the comb spacing $(48.9 \mathrm{GHz} / 2=24.45 \mathrm{GHz})$.

For arbitrary RF waveform generation, the soliton crystal micro-comb was flattened and modulated with the RF input pulse in order to multicast the RF waveform onto all of the wavelength channels to yield 81 replicas. The second WaveShaper accurately shaped the comb power according to the designed weights. The wavelength channels for positive and negative taps were separately measured by an optical spectrum analyzer. Finally, the delayed replicas were combined and then summed upon photodetection. By tailoring the comb lines' power according to the tap weights, arbitrary waveform generation could be achieved.

\section{Experimental Results}

The experimental setup is shown in Fig. 1(c). A CW laser is amplified and with its polarization state adjusted to pump a nonlinear high Q factor Hydex MRR, which featured a Q factor of over 1.5 million and a free spectral range of $\sim 0.4 \mathrm{~nm}$, or $48.9 \mathrm{GHz}$. As the detuning between the pump laser and the MRR changed, dynamic parametric oscillation states corresponding to distinctive solutions of the LugiatoLefever equation were initiated [44]. We generated soliton crystal micro-combs [45], which were tightly packed solitons circulating in the MRR as a result of a mode crossing (at $~ 1552 \mathrm{~nm}$ in our case), and manifested by the generated distinctive palm-like comb spectrum (Figure 1(b)).

For phase-encoded signal generation, 60 lines of the micro-comb were flattened, using two stages of WaveShapers (Finisar 4000S) to acquire a high link gain and signal-to-noise ratio. This was achieved by pre-flattening the micro-comb lines with the first WaveShaper (WS1) such that the optical power distribution of the wavelength channels roughly matched with the desired channel weights. The second WaveShaper was employed for accurate comb shaping assisted by a feedback loop as well as to separate the wavelength channels into two parts (port 1 and port 2 of the WaveShaper) according to the polarity of the designed binary phase codes. The feedback loop was constructed by reading the optical 
spectrum with an optical spectrum analyzer and comparing with designed weights to generate an error signal, which was fed back into the second WaveShaper (WS2) to calibrate its loss until the error was below $0.2 \mathrm{~dB}$. Here, we use a Gaussian pulse with a duration of $\Delta t=84 \mathrm{ps}$, as the RF fragment $f[t]$. The input RF pulse was imprinted onto the comb lines, generating replicas across all the wavelength channels. The replicas then went through a $\sim 13 \mathrm{~km}$ long spool of standard single mode fiber to progressively delay them, leading to a delay step of $\sim 84$ ps between the adjacent wavelength channels that matched with the duration of the RF pulse $\Delta t$. Finally, the wavelength channels were separated into two parts according to designed phase codes and sent to a balanced photodetector (Finisar, $40 \mathrm{GHz}$ ) to achieve negative and positive replicas for the phase encoding.

By applying designed phase codes during the separation of the wavelength channels, the sine cycles could be $\pi$-phase shifted at desired times. The phase-encoded results are shown in Figure 2. The number of Gaussian pulses for each RF segment (denoted by $m$ ) was reconfigured from 6 to 2, corresponding toreconfigurable sequence lengths $(N / m)$ ranging from 10 to 30 and phase coding speeds $1 /(m \Delta t)$ ranging from 1.98 to $5.95 \mathrm{~Gb} / \mathrm{s}$. The employed phase codes were denoted both by the shaded areas and the stair waveforms (black solid line). This result shows that our photonic phase coder can offer reconfigurable sequence lengths to address the performance tradeoffs between range resolution and system complexity. To acquire a large pulse compression ratio for a high resolution, the sequence length should be maximized, where the number of Gaussian pulses for each RF segment $(m)$ should be set to 2 . Further, to reduce the complexity and cost of the RF system (such as the number of range gates at the receiver), the sequence length could be reduced by either employing fewer wavelength channels or by increasing $m$. The corresponding optical spectra (Figure 2(a, c, e)) were measured at the output of WaveShaper to show the positive and negative phase codes realized by changing the wavelength channels' output ports at the WaveShaper. The encoded RF waveforms (Figure 2(b, $d, f)$ ) clearly show the flipped phase of the RF segments at the time of negative phase codes, where the number of sine cycles was reconfigured as well according to $\mathrm{m}$. This result also shows that our approach is fully reconfigurable for different phase codes and encoding speeds. We note that higher encoding speeds can be achieved by reducing the duration of the RF fragment and the delay step $\Delta t$.

We also calculated the autocorrelation (Figure 3(a)) of the phase-encoded RF waveforms. As the sequence length varied from 10 to 30 , the full width at half-maximum (FWHM) of the compressed pulses varied from 0.52 to $0.17 \mathrm{~ns}$, which corresponds to a pulse suppression ratio rangingfrom 9.7 to 29.6. Meanwhile the peak-to-sidelobe ratio (PSR) also increased with the sequence length from 4.17 to $6.59 \mathrm{~dB}$. These results confirm that the pulse compression ratio of an RF phase-encoded signal is linearly related to its sequence length [46], and that this can be significantly enhanced with our approach by using a larger number of wavelength channels of the microcomb. Figure 3(b) shows calculated examples of the estimated outputs of the range gates, with different distances. Considering an example with a sequence length $N / m=30$, the delay of the matched filters would be $2 \Delta t=168 \mathrm{ps}$. The tap coefficients for the $I_{\text {th }}$ matched filter are $c[k-1$, where $c[k], k=1,2, \ldots 30$, is the employed phase codes. The range resolution of the radar, which is the minimum distance between two resolvable targets, is determined by the delay step 
$(2 \Delta t)$ of the matched filters, which is given by $2 \Delta t \cdot c=5 \mathrm{~cm}$, where $c=3 \times 10^{8} \mathrm{~m} / \mathrm{s}$ is the speed of RF signals in air. If the distance between the target and radar is $R$, then the delay would be $2 R / c$. The range gates would have a maximum output at the lth range gate, $I=2 R /(c 2 \Delta t)$. We note here that this calculation only shows the basic relations between our phase encoder and the radar systems' performance. Practical radar systems are subject to more complicated trade-offs involving capability versus performance.

For arbitrary waveform generation, 81 lines of the micro-comb were flattened. On the basis of phase encoding, by tailoring the comb lines' power according to the tap weights arbitrary waveform generation could be achieved. To demonstrate the flexibility of our photonic RF signal generation approach, we designed square waveforms (Figure $4(a-\varangle)$ ) with a tunable duty cycle ratio ranging from $10-90 \%$. Similarly, sawtooth waveforms (Fig. 4(a- $₫)$ ) with a tunable slope ranging from 0.2 to 1 were generated. The received signals were digitally sampled by an $80 \mathrm{GSa} / \mathrm{s}$ real-time oscilloscope, with the measured waveforms normalized to the peak intensity. We then demonstrated the frequency-modulated waveform, as shown in Figure 4(b), for which the sign of the frequency modulation (or 'chirp') can be programmed to sweep from high to low and then from low to high frequency, which is very difficult to achieve with electronic techniques. We compared the experimental results obtained with the corresponding calculated instantaneous frequency of the designed symmetric concave quadratic chirp, both of which are shown in Figure $4(\mathrm{~b}-\mathrm{Q}, \mathrm{Z})$.

Compared to electronic means of arbitrary waveform generation, our scheme makes possible RF waveforms with much higher instantaneous bandwidth by simply shortening the time delay and the corresponding optical pulse width. Note that we used a commercial arbitrary waveform generator (Keysight, $65 \mathrm{GSa} / \mathrm{s}$ ) here to generate the pulse for this proof of principle demonstration. This allowed us to investigate the device performance by, for example, readily changing the pulse width to test the capability of our system to span different frequency ranges for the RF waveform. In practice, however, electronic AWGs are not necessary and can easily be replaced with many other readily available approaches that are simpler, easier and cheaper [47].

The quality and stability of the soliton crystal comb was more than good enough for our experiments. Indeed, the soliton crystal combs have been shown to be stable and reliable enough to support ultrahigh bandwidth communications at 44Terabits/s [48], with extremely high stability over many 10's of hours. The energy efficiency and noise of our system would be increased further by reducing the loss through the system, for example by achieving higher levels of integration.

There is significant potential for substantially higher levels of integration than the discrete devices used here - ultimately achieving fully monolithically integrated embodiments of our system. The central component of our system, the optical frequency comb source, is already integrated. Further, all of the other components have been demonstrated in integrated form, including on-chip InP spectral shapers [49], highspeed integrated lithium niobate modulators [50], integrated dispersive elements [51], and photodetectors [52]. Finally, many recent advances have been made in reducint the power-consumption 
of Kerr combs [53] that would greatly reduce the system energy requirements. These results will have a significant impact on the field of microwave photonics, [54-92] particularly that based on micro-combs [93-209] with potential applications even to the mid IR [210-216]

\section{Conclusion}

We demonstrate photonic RF phase-encoded signal generation and RF arbitrary waveform generator using an integrated micro-comb source. A single-cycle Gaussian pulse was multicast onto the comb wavelengths to assemble the desired phase-encoded RF waveform and RF arbitrary waveform. For RF phase encoding, a high pulse compression ratio of 29.6 and phase encoding speed of $5.95 \mathrm{~Gb} / \mathrm{s}$ was achieved, enabled by the use of 60 wavelengths generated by the microcomb. The sequence length was reconfigured by adjusting the length of each phase code, which led to a reconfigurable encoding speed. For RF arbitrary waveform generation, the comb source provides over 80 channels, that we use to successfully achieve arbitrary waveform shapes including square waveforms with a tunable duty ratio ranging from $10-90 \%$, sawtooth waveforms with a tunable slope ratio of 0.2 to 1 , and a symmetric concave quadratic chirp waveform with an instantaneous frequency of sub- $\mathrm{GHz}$. These results verify that our approach to high-speed RF phase encoding and user-defined AWG is competitive in terms of performance, with potentially lower cost and footprint.

\section{Declarations}

Competing interests: The authors declare no competing interests.

\section{References}

1. Ghelfi, P., Laghezza, F., Scotti, F., Serafino, G., Capria, A., Pinna, S., Onori, D., Porzi, C., Scaffardi, M., Malacarne, A., Vercesi, V., Lazzeri, E., Berizzi, F., and Bogoni, A.: 'A fully photonics-based coherent radar system', Nature, 2014, 507, (7492), pp. 341-345

2. Skolnik, M.: 'Role of radar in microwaves', IEEE Transactions on Microwave Theory and Techniques, 2002, 50, (3), pp. 625-632

3. Chou, J., Han, Y., and Jalali, B.: 'Adaptive RF-photonic arbitrary waveform generator', IEEE Photonics Technology Letters, 2003, 15, (4), pp. 581-583

4. Cundiff, S.T., and Weiner, A.M.: 'Optical arbitrary waveform generation', Nat. Photonics, 2010, 4, (11), pp. 760-766

5. Rashidinejad, A., Li, Y., and Weiner, A.M.: 'Recent Advances in Programmable Photonic-Assisted Ultrabroadband Radio-Frequency Arbitrary Waveform Generation', IEEE Journal of Quantum Electronics, 2016, 52, (1), pp. 1-17

6. Ghelfi, P., Scotti, F., Laghezza, F., and Bogoni, A.: 'Photonic Generation of Phase-Modulated RF Signals for Pulse Compression Techniques in Coherent Radars', Journal of Lightwave Technology, 2012, 30, (11), pp. 1638-1644 
7. Capmany, J., and Novak, D.: 'Microwave photonics combines two worlds', Nat. Photonics, 2007, 1, (6), pp. 319-330

8. Khan, M.H., Shen, H., Xuan, Y., Zhao, L., Xiao, S., Leaird, D.E., Weiner, A.M., and Qi, M.: 'Ultrabroadbandwidth arbitrary radiofrequency waveform generation with a silicon photonic chip-based spectral shaper', Nat. Photonics, 2010, 4, (2), pp. 117-122

9. Chi, H., and Yao, J.: 'Photonic Generation of Phase-Coded Millimeter-Wave Signal Using a Polarization Modulator', IEEE Microwave and Wireless Components Letters, 2008, 18, (5), pp. 371373

10. Zhang, Y., and Pan, S.: 'Generation of phase-coded microwave signals using a polarizationmodulator-based photonic microwave phase shifter', Opt. Lett., 2013, 38, (5), pp. 766-768

11. Zhu, S., Shi, Z., Li, M., Zhu, N.H., and Li, W.: 'Simultaneous frequency upconversion and phase coding of a radio-frequency signal for photonic radars', Opt. Lett., 2018, 43, (3), pp. 583-586

12. Zhu, S., Li, M., Wang, X., Zhu, N.H., Cao, Z.Z., and Li, W.: 'Photonic generation of background-free binary phase-coded microwave pulses', Opt. Lett., 2019, 44, (1), pp. 94-97

13. Li, Z., Li, W., Chi, H., Zhang, X., and Yao, J.: 'Photonic generation of phase-coded microwave signal with large frequency tunability', IEEE Photonics Technology Letters, 2011, 23, (11), pp. 712-714

14. Liu, W., and Yao, J.: 'Photonic generation of microwave waveforms based on a polarization modulator in a Sagnac loop', Journal of Lightwave Technology, 2014, 32, (20), pp. 3637-3644

15. Wang, J., Shen, H., Fan, L., Wu, R., Niu, B., Varghese, L.T., Xuan, Y., Leaird, D.E., Wang, X., and Gan, F.: 'Reconfigurable radio-frequency arbitrary waveforms synthesized in a silicon photonic chip', Nat. Commun., 2015, 6, (1), pp. 1-8

16. Ashrafi, R., Li, M., and Azaña, J.: 'Multi-TBaud optical coding based on superluminal space-to-time mapping in long period gratings', Optics and Photonics Journal, 2013, 3, (2), pp. 126-130

17. Rashidinejad, A., and Weiner, A.M.: 'Photonic radio-frequency arbitrary waveform generation with maximal time-bandwidth product capability', Journal of Lightwave Technology, 2014, 32, (20), pp. 3383-3393

18. Jiang, Z., Huang, C.-B., Leaird, D.E., and Weiner, A.M.: 'Optical arbitrary waveform processing of more than 100 spectral comb lines', Nat. Photonics, 2007, 1, (8), pp. 463-467

19. Dai, Y., and Yao, J.: 'Microwave pulse phase encoding using a photonic microwave delay-line filter', Opt. Lett., 2007, 32, (24), pp. 3486-3488

20. Wu, J., Xu, X., Nguyen, T.G., Chu, S.T., Little, B.E., Morandotti, R., Mitchell, A., and Moss, D.J.: 'RF Photonics: An Optical Microcombs' Perspective', IEEE Journal of Selected Topics in Quantum Electronics, 2018, 24, (4), pp. 1-20

21. Xu, X., Tan, M., Wu, J., Nguyen, T.G., Chu, S.T., Little, B.E., Morandotti, R., Mitchell, A., and Moss, D.J.: 'High performance RF filters via bandwidth scaling with Kerr micro-combs', APL Phontonics, 2019, 4, (2), pp. 026102 
22. Xu, X., Tan, M., Wu, J., Nguyen, T.G., Chu, S.T., Little, B.E., Morandotti, R., Mitchell, A., and Moss, D.J.: 'High performance RF filters via bandwidth scaling with Kerr micro-combs', APL Phontonics, 2019, 4, (2), pp. 8

23. Xu, X., Wu, J., Shoeiby, M., Nguyen, T.G., Chu, S.T., Little, B.E., Morandotti, R., Mitchell, A., and Moss, D.J.: 'Reconfigurable broadband microwave photonic intensity differentiator based on an integrated optical frequency comb source', APL Phontonics, 2017, 2, (9), pp. 096104

24. Tan, M., Xu, X., Corcoran, B., Wu, J., Boes, A., Nguyen, T.G., Chu, S.T., Little, B.E., Morandotti, R., Mitchell, A., and Moss, D.J.: 'Microwave and RF Photonic Fractional Hilbert Transformer Based on a $50 \mathrm{GHz}$ Kerr Micro-Comb', Journal of Lightwave Technology, 2019, 37, (24), pp. 6097-6104

25. Tan, M., Xu, X., Corcoran, B., Wu, J., Boes, A., Nguyen, T.G., Chu, S.T., Little, B.E., Morandotti, R., Mitchell, A., and Moss, D.J.: 'RF and Microwave Fractional Differentiator Based on Photonics', IEEE Trans. Circuits Syst. II-Express Briefs, 2020, 67, (11), pp. 2767-2771

26. Xu, X., Tan, M., Wu, J., Boes, A., Corcoran, B., Nguyen, T.G., Chu, S.T., Little, B.E., Morandotti, R., Mitchell, A., and Moss, D.J.: 'Photonic RF and Microwave Integrator Based on a Transversal Filter With Soliton Crystal Microcombs', IEEE Trans. Circuits Syst. II-Express Briefs, 2020, 67, (12), pp. 3582-3586

27. Tan, M., Xu, X., Boes, A., Corcoran, B., Wu, J., Nguyen, T.G., Chu, S.T., Little, B.E., Lowery, A.J., Morandotti, R., Mitchell, A., and Moss, D.J.: 'Highly Versatile Broadband RF Photonic Fractional Hilbert Transformer Based on a Kerr Soliton Crystal Microcomb', Journal of Lightwave Technology, 2021, 39, (24), pp. 7581-7587

28. Xu, X., Wu, J., Tan, M., Nguyen, T.G., Chu, S.T., Little, B.E., Morandotti, R., Mitchell, A., and Moss, D.J.: 'Broadband Microwave Frequency Conversion Based on an Integrated Optical Micro-Comb Source', Journal of Lightwave Technology, 2020, 38, (2), pp. 332-338

29. Xu, X., Wu, J., Nguyen, T.G., Moein, T., Chu, S.T., Little, B.E., Morandotti, R., Mitchell, A., and Moss, D.J.: 'Photonic microwave true time delays for phased array antennas using a $49 \mathrm{GHz}$ FSR integrated optical micro-comb source Invited', Photonics Res., 2018, 6, (5), pp. B30-B36

30. Xu, X., Wu, J., Nguyen, T. Chu, S.T., Little, B.E., Morandotti, R., Mitchell, A., and Moss, D.J.: 'Broadband RF Channelizer Based on an Integrated Optical Frequency Kerr Comb Source', Journal of Lightwave Technology, 2018, 36, (19), pp. 4519-4526

31. Xu, X., Tan, M., Wu, J., Boes, A., Nguyen, T.G., Chu, S.T., Little, B.E., Morandotti, R., Mitchell, A., and Moss, D.J.: 'Broadband Photonic RF Channelizer With 92 Channels Based on a Soliton Crystal Microcomb', Journal of Lightwave Technology, 2020, 38, (18), pp. 5116-5121

32. Xu, X., Tan, M., Wu, J., Boes, A., Corcoran, B., Nguyen, T.G., Chu, S.T., Little, B.E., Morandotti, R., Mitchell, A., and Moss, D.J.: 'Photonic RF Phase-Encoded Signal Generation With a Microcomb Source', Journal of Lightwave Technology, 2020, 38, (7), pp. 1722-1727

33. Tan, M., Xu, X., Boes, A., Corcoran, B., Wu, J., Nguyen, T.G., Chu, S.T., Little, B.E., Morandotti, R., Mitchell, A., and Moss, D.J.: 'Photonic RF Arbitrary Waveform Generator Based on a Soliton Crystal Micro-Comb Source', Journal of Lightwave Technology, 2020, 38, (22), pp. 6221-6226 
34. X. Xu, M. Tan, B. Corcoran, J. Wu, A. Boes, T. G. Nguyen, S. T. Chu, B. E. Little, D. G. Hicks, R. Morandotti, A. Mitchell, and D. J. Moss, "11 TOPs photonic convolutional accelerator for optical neural networks", Nature Vol. 589 (7840) 44-51 (2021).

35. M. Tan, X. Xu, J. Wu, R. Morandotti, A. Mitchell, and D. J. Moss, "RF and microwave photonic temporal signal processing with Kerr micro-combs", Advances in Physics X, VOL. 6, NO. 1, 1838946 (2021).

36. M. Tan, X. Xu, J. Wu, T. G. Nguyen, S. T. Chu, B. E. Little, R. Morandotti, A. Mitchell, and D. J. Moss, “Photonic Radio Frequency Channelizers based on Kerr Optical Micro-combs", IOP Journal of Semiconductors Vol. 42 (4), 041302 (2021).

37. M. Tan, X. Xu, J. Wu, T. G. Nguyen, S. T. Chu, B. E. Little, R. Morandotti, A. Mitchell, and D. J. Moss, "Orthogonally polarized Photonic Radio Frequency single sideband generation with integrated microring resonators", IOP Journal of Semiconductors, Vol. 42 (4), 041305 (2021).

38. M. Tan, X. Xu, J. Wu, Bill Corcoran, A. Boes, T. G. Nguyen, S. T. Chu, B. E. Little, R. Morandotti, A. Lowery, A. Mitchell, and D. J. Moss, "'Highly Versatile Broadband RF Photonic Fractional Hilbert Transformer Based on a Kerr Soliton Crystal Microcomb”, Journal of Lightwave Technology Vol. 39 (24) 7581-7587 (2021).

39. Chawaphon Prayoonyong, Andreas Boes, Xingyuan Xu, Mengxi Tan, Sai T. Chu, Brent E. Little, Roberto Morandotti, Arnan Mitchell, David J. Moss, and Bill Corcoran, "Frequency comb distillation for optical superchannel transmission”, Journal of Lightwave Technology Vol. 39 (23) 7383-7392 (2021).

40. Mengxi Tan, Xingyuan Xu, Jiayang Wu, Bill Corcoran, Andreas Boes, Thach G. Nguyen, Sai T. Chu, Brent E. Little, Roberto Morandotti, Arnan Mitchell, and David J. Moss, "Integral order photonic RF signal processors based on a soliton crystal micro-comb source”, IOP Journal of Optics Vol. 23 (11) 125701 (2021).

41. M. Tan, X. Xu, J. Wu, R. Morandotti., A. Mitchell, and D. J. Moss, "Photonic RF and microwave filters based on 49GHz and 200GHz Kerr microcombs", Optics Communications Vol. 465, Article: 125563 (2020).

42. X. Xu, M. Tan, J. Wu, S. T. Chu, B. E. Little, R. Morandotti, A. Mitchell, B. Corcoran, D. Hicks, and D. J. Moss, "Photonic perceptron based on a Kerr microcomb for scalable high speed optical neural networks", Laser and Photonics Reviews Vol. 14 (8) 2000070 (2020).

43. X. Xu, M. Tan, J. Wu, R. Morandotti, A. Mitchell, and D. J. Moss, “Microcomb-based photonic RF signal processing”, IEEE Photonics Technology Letters Vol. 31 (23) 1854-1857 (2019).

44. Pasquazi, A., Peccianti, M., Razzari, L., Moss, D.J., Coen, S., Erkintalo, M., Chembo, Y.K., Hansson, T., Wabnitz, S., and Del'Haye, P.: 'Micro-combs: A novel generation of optical sources', Physics Reports, 2018, 729, pp. 1-81

45. Cole, D.C., Lamb, E.S., Del'Haye, P., Diddams, S.A., and Papp, S.B.: 'Soliton crystals in Kerr resonators', Nat. Photonics, 2017, 11, (10), pp. 671-676 
46. Levanon, N., and Getz, B.: 'Comparison between linear FM and phase-coded CW radars', IEE Proceedings-Radar, Sonar and Navigation, 1994, 141, (4), pp. 230-240

47. Torres-Company, V., and Weiner, A.M.: 'Optical frequency comb technology for ultra-broadband radiofrequency photonics', Laser Photon. Rev., 2014, 8, (3), pp. 368-393

48. Corcoran, B., Tan, M., Xu, X., Boes, A., Wu, J., Nguyen, T.G., Chu, S.T., Little, B.E., Morandotti, R., and Mitchell, A., and Moss, D.J.: 'Ultra-dense optical data transmission over standard fibre with a single chip source', Nat. Commun., 2020, 11, (1), pp.1-7

49. Metcalf, A.J., Kim, H.-J., Leaird, D.E., Jaramillo-Villegas, J.A., McKinzie, K.A., Lal, V., Hosseini, A., Hoefler, G.E., Kish, F., and Weiner, A.M.: 'Integrated line-by-line optical pulse shaper for high-fidelity and rapidly reconfigurable RF-filtering', Opt. Express, 2016, 24, (21), pp. 23925-23940

50. Wang, C., Zhang, M., Chen, X., Bertrand, M., Shams-Ansari, A., Chandrasekhar, S., Winzer, P., and Lončar, M.: 'Integrated lithium niobate electro-optic modulators operating at CMOS-compatible voltages', Nature, 2018, 562, (7725), pp.101-104

51. Molony, A., Lin, Z., Williams, J.A.R., Bennion, I., Edge, C., and Fells, J.: 'Fiber Bragg-grating true timedelay systems: discrete-grating array 3-b delay lines and chirped-grating 6-b delay lines', IEEE Transactions on Microwave Theory and Techniques, 1997, 45, (8), pp. 1527-1530

52. Michel, J., Liu, J., and Kimerling, L.C.: 'High-performance Ge-on-Si photodetectors', Nat. Photonics, 2010, 4, (8), pp. 527-534

53. Stern, B., Ji, X., Okawachi, Y., Gaeta, A.L., and Lipson, M.: 'Battery-operated integrated frequency comb generator', Nature, 2018, 562, (7727), pp. 401-405

54. L. Moura, "Radio Frequency Implementation of the Fractional Hilbert Transform with Transversal Filters," Circuits, Systems \& Signal Processing., vol. 26, no. 3, pp. 407-417, Jun. 2007.

55. A. W. Lohmann, D. Mendlovic, and Z. Zalevsky, “Fractional Hilbert transform,” Opt. Lett., vol. 21, no. 4, pp. 281-283, Feb. 1996.

56. J. A. Davis, D. E. McNamara, and D. M. Cottrell, "Analysis of the fractional Hilbert transform," Appl. Opt., vol. 37, no. 29, pp. 6911-6913, Oct. 1998.

57. C. D. Holdenried, J. W. Haslett, and B. Davies, "A fully integrated 10-Gb/s tapped delay Hilbert transformer for optical single sideband," IEEE Microw. Wireless Compon. Lett., vol. 15, no. 5, pp. 303305, May 2005.

58. H. Emami, N. Sarkhosh, L. A. Bui, and A. Mitchell, "Wideband RF photonic in-phase and quadraturephase generation," Opt. Lett., vol. 33, no. 2, pp. 98-100, Jan 15. 2008.

59. M. Li, and J. P. Yao, "All-fiber temporal photonic fractional Hilbert transformer based on a directly designed fiber Bragg grating," Opt. Lett., vol. 35, no. 2, pp. 223-225, Jan. 2010.

60. M. Li, and J. P. Yao, "Experimental Demonstration of a Wideband Photonic Temporal Hilbert Transformer Based on a Single Fiber Bragg Grating," IEEE Photon. Technol. Lett., vol. 22, no. 21 pp. 1559-1561, Nov. 2010. 
61. M. H. Asghari, and J. Azana, "All-optical Hilbert transformer based on a single phase-shifted fiber Bragg grating: design and analysis," Opt. Lett., vol. 34, no. 3, pp. 334-336, Feb. 2009.

62. T. Yang, J. Dong, L. Liu, S. Liao, S. Tan, L. Shi, D. Gao, and X. Zhang, "Experimental observation of optical differentiation and optical Hilbert transformation using a single SOI microdisk chip," Sci. Rep., vol. 4, pp. 3960, 2014.

63. W. Liu, et al., "A Fully Reconfigurable Photonic Integrated Signal Processor," Nature Photonics, vol. 10, no. 3, pp. 190-196, 2016.

64. Z. Zhang, et al., "Wideband and continuously-tunable fractional photonic Hilbert transformer based on a single high-birefringence planar Bragg grating," Optics Express, vol. 26, pp. 20450-20458, 2018.

65. F. Zeng, and J. Yao, "An Approach to Ultrawideband Pulse Generation and Distribution Over Optical Fiber," IEEE Photonics Technol. Lett., vol. 18, no. 7, pp. 823-825, Apr. 2006.

66. S. Pan, and J. Yao, "Optical generation of polarity- and shape-switchable ultrawideband pulses using a chirped intensity modulator and a first-order asymmetric Mach-Zehnder interferometer," Opt. Lett., vol. 34, no. 9, pp. 1312-1314, May. 2009.

67. Y. Yu, J. Dong, X. Li, and X. Zhang, "Ultra-Wideband Generation Based on Cascaded Mach-Zehnder Modulators," IEEE Photonics Technol. Lett., vol. 23, no. 23, Dec. 2011.

68. L. Zhuang, M. R. Khan, W. Beeker, A. Leinse, R. Heideman, and C. Roeloffzen, "Novel microwave photonic fractional Hilbert transformer using a ring resonator-based optical all-pass filter," Opt. Exp., vol. 20, no. 24, pp. 26499-26510, Nov. 2012.

69. C. Sima, J. C. Gates, C. Holmes, P. L. Mennea, M. N. Zervas, and P. G. R. Smith, "Terahertz bandwidth photonic Hilbert transformers based on synthesized planar Bragg grating fabrication," Opt. Lett., vol. 38, no. 17, pp. 3448-3451, Sep. 2013.

70. H. Shahoei, P. Dumais, and J. P. Yao, "Continuously tunable photonic fractional Hilbert transformer using a high-contrast germanium-doped silica-on-silicon microring resonator," Opt. Lett., vol. 39, no. 9, pp. 2778-2781, May 2014.

71. Z. Li, Y. Han, H. Chi, X. Zhang, and J. P. Yao, "A continuously Tunable Microwave Fractional Hilbert Transformer Based on a Nonuniformly Spaced Photonic Microwave Delay-Line Filter," J. Lightwave Technol., vol. 30, no. 12, pp. 1948-1953, Jun. 2012.

72. Z. Li, H. Chi, X. Zhang, and J. P. Yao, "A Continuously Tunable Microwave Fractional Hilbert Transformer Based on a Photonic Microwave Delay-Line Filter Using a Polarization Modulator," IEEE Photon. Technol. Lett., vol. 23, no. 22, pp. 1694-1696, Nov. 2011.

73. A. O.-Blanch, J. Mora, J. Capmany, B. Ortega, and D. Pastor, "Tunable radio-frequency photonic filter based on an actively mode-locked fiber laser," Opt. Lett., vol. 31, no. 6, pp. 709-711, Mar. 2006.

74. V. R. Supradeepa, C. M. Long, R. Wu. F. Ferdous, E. Hamidi, D. E. Leaird, and A. M. Weiner, "Combbased radiofrequency photonic filters with rapid tunability and high selectivity," Nature Photonics, vol. 6, pp. 186-194, Mar. 2012.

75. V. T.-Company, and A. M. Weiner, "Optical frequency comb technology for ultra-broadband radiofrequency photonics," Laser Photonics Rev., vol. 8, no. 3, pp. 368-393, 2014. 
76. S. Pan, J. Yao, "Optical generation of polarity- and shape-switchable ultrawideband pulses using a chirped intensity modulator and a first-order asymmetric Mach-Zehnder interferometer," Opt. Lett., vol. 34, no. 9, pp. 1312-1314, 2009.

77. X. Li, J. Dong, Y. Yu, and X. Zhang, "A Tunable Microwave Photonic Filter Based on an All-Optical Differentiator," IEEE Photon. Technol. Lett., vol. 23, no. 22, pp. 308-310, Mar. 2011.

78. Y. Han, Z. Li, and J. Yao, "A Microwave Bandpass Differentiator Implemented Based on a Nonuniformly-Spaced Photonic Microwave Delay-Line Filter," J. Lightw. Technol., vol. 29, no. 22, pp. 3470-3475, Nov. 2011.

79. R. Ashrafi and J. Azaña, "Figure of merit for photonic differentiators," Opt. Exp., vol. 20, no. 3, pp. 2626-2639, Jan. 2012.

80. F. Zeng and J. Yao, "Ultrawideband Impulse Radio Signal Generation Using a High-Speed Electrooptic Phase Modulator and a Fiber-Bragg-Grating-Based Frequency Discriminator," IEEE Photon. Technol. Lett., vol. 18, no. 19, pp. 2062-2064, Oct. 2006.

81. P. Li, H. Chen, M. Chen, and S. Xie, “Gigabit/s Photonic Generation, Modulation, and Transmission for a Reconfigurable Impulse Radio UWB Over Fiber System," IEEE Photon. Technol. Lett., vol. 4, no. 3, pp. 805-816, Jun. 2012.

82. Y. Yu, F. Jiang, H. Tang, L. Xu, X. Liu, J. Dong, and X. Zhang, "Reconfigurable photonic temporal differentiator based on a dual-drive Mach-Zehnder modulator," Opt. Exp., vol. 24, no. 11, pp. 1173911748, May 2016.

83. P. Velanas, A. Bogris, A. Argyris, and D. Syvridis, "High-Speed All-Optical First- and Second-Order Differentiators Based on Cross-Phase Modulation in Fibers," J. Lightw. Technol., vol. 26, no. 18, pp. 3269-3276, Sep. 2008.

84. J. Xu, X. Zhang, J. Dong, D. Liu, and D. Huang, "All-optical differentiator based on cross-gain modulation in semiconductor optical amplifier," Opt. Lett., vol. 32, no. 20, pp. 3029-3031, Oct. 2007.

85. J. Xu, X. Zhang, J. Dong, D. Liu, and D. Huang, "High-speed all-optical differentiator based on a semiconductor optical amplifier and an optical filter," Opt. Lett., vol. 32, no. 13, pp. 1872-1874, Jul. 2007.

86. F. Wang, J. Dong, E. Xu, and X. Zhang, "All-optical UWB generation and modulation using SOA-XPM effect and DWDM-based multi-channel frequency discrimination," Opt. Exp., vol. 18, no. 24, pp. 24588-24594, Nov. 2010.

87. V. Moreno, M. Rius, J. Mora, M. A. Muriel, and J. Capmany, "Integrable high order UWB pulse photonic generator based on cross phase modulation in a SOA-MZI," Opt. Exp., vol. 21, no. 19, pp. 2291122917, Sep. 2013.

88. Q. Wang and J. Yao, "Switchable optical UWB monocycle and doublet generation using a reconfigurable photonic microwave delay-line filter," Opt. Exp., vol. 15, no. 22, pp. 14667-14672, Oct. 2007.

89. M. Bolea, J. Mora, B. Ortega, and J. Capmany, "Optical UWB pulse generator using an Ntap microwave photonic filter and phase inversion adaptable to different pulse modulation formats," Opt. 
Exp., vol. 17, no. 7, pp. 5023-50332, Mar. 2009.

90. B. Mathieu, P. Melchior, A. Oustaloup, C. Ceyral, “Fractional differentiation for edge detection,” Signal Processing, vol. 83, pp. 2421-2432, Nov. 2003.

91. A. Oustaloup, F. Levron, B. Mathieu, and F. M. Nanot, "Frequency-Band Complex Noninteger Differentiator: Characterization and Synthesis," IEEE Trans. on Circuit and Systems - I: Fundamental Theory and Application, vol. 47, no. 1, pp. 25-39, Jan. 2000.

92. F. Li, Y. Park, and J. Azaña, “Linear Characterization of Optical Pulses With Durations Ranging From the Picosecond to the Nanosecond Regime Using Ultrafast Photonic Differentiation," J. Lightw. Technol., vol. 27, no. 1, pp. 4623-4633, 2009.

93. M. Tan, X. Xu, J. Wu, T. G. Nguyen, S. T. Chu, B. E. Little, R. Morandotti, A. Mitchell, and D. J. Moss, "Photonic Radio Frequency Channelizers based on Kerr Optical Micro-combs", IOP Journal of Semiconductors Vol. 42 (4), 041302 (2021).

94. M. Tan, X. Xu, J. Wu, T. G. Nguyen, S. T. Chu, B. E. Little, R. Morandotti, A. Mitchell, and D. J. Moss, “Orthogonally polarized Photonic Radio Frequency single sideband generation with integrated microring resonators", IOP Journal of Semiconductors, Vol. 42 (4), 041305 (2021).

95. C.Prayoonyong et al., "Frequency comb distillation for optical superchannel transmission”, Journal of Lightwave Technology Vol. 39 (23) 7383-7392 (2021).

96. X. Xu et al, "Photonic perceptron based on a Kerr microcomb for scalable high speed optical neural networks", Laser and Photonics Reviews, vol. 14, no. 8, 2000070 (2020). DOI:

10.1002/Ipor.202000070.

97. X. Xu, et al., "11 TOPs photonic convolutional accelerator for optical neural networks", Nature vol. 589, pp. 44-51 (2021).

98. Pasquazi, A. et al. Micro-combs: a novel generation of optical sources. Physics Reports 729, 1-81 (2018).

99. Moss, D. J. et al., "New CMOS-compatible platforms based on silicon nitride and Hydex for nonlinear optics", Nature photonics 7, 597 (2013).

100. B. Corcoran, et al., "Ultra-dense optical data transmission over standard fiber with a single chip source", Nature Communications, vol. 11, Article:2568, 2020.

101. Kues, M. et al. Quantum optical microcombs. Nature Photonics 13, (3) 170-179 (2019). doi:10.1038/s41566-019-0363-0

102. C. Reimer, L. Caspani, M. Clerici, et al., "Integrated frequency comb source of heralded single photons," Optics Express, vol. 22, no. 6, pp. 6535-6546, 2014.

103. C. Reimer, et al., "Cross-polarized photon-pair generation and bi-chromatically pumped optical parametric oscillation on a chip", Nature Communications, vol. 6, Article 8236, 2015. DOI: $10.1038 /$ ncomms9236.

104. L. Caspani, C. Reimer, M. Kues, et al., "Multifrequency sources of quantum correlated photon pairs on-chip: a path toward integrated Quantum Frequency Combs," Nanophotonics, vol. 5, no. 2, 
pp. 351-362, 2016.

105. C. Reimer et al., "Generation of multiphoton entangled quantum states by means of integrated frequency combs," Science, vol. 351, no. 6278, pp. 1176-1180, 2016.

106. M. Kues, et al., "On-chip generation of high-dimensional entangled quantum states and their coherent control”, Nature, vol. 546, no. 7660, pp. 622-626, 2017.

107. P. Roztocki et al., "Practical system for the generation of pulsed quantum frequency combs," Optics Express, vol. 25, no. 16, pp. 18940-18949, 2017.

108. Y. Zhang, et al., "Induced photon correlations through superposition of two four-wave mixing processes in integrated cavities", Laser and Photonics Reviews, vol. 14, no. 7, pp. 2000128, 2020. DOI: 10.1002/Ipor.202000128

109. C. Reimer, et al.,'High-dimensional one-way quantum processing implemented on d-level cluster states", Nature Physics, vol. 15, no.2, pp. 148-153, 2019.

110. Stern, B., Ji, X., Okawachi, Y., Gaeta, A. L. \& Lipson, M. Battery-operated integrated frequency comb generator. Nature 562, 401 (2018).

111. H. Bao, et al., Laser cavity-soliton microcombs, Nature Photonics, vol. 13, no. 6, pp. 384-389, Jun. 2019.

112. Bao, C., et al., Direct soliton generation in microresonators, Opt. Lett, 42, 2519 (2017).

113. M.Ferrera et al., "CMOS compatible integrated all-optical RF spectrum analyzer", Optics Express, vol. 22, no. 18, 21488-21498 (2014).

114. A. Pasquazi, et al., "Sub-picosecond phase-sensitive optical pulse characterization on a chip", Nature Photonics, vol. 5, no. 10, pp. 618-623 (2011).

115. M. Kues, et al., "Passively modelocked laser with an ultra-narrow spectral width", Nature Photonics, vol. 11, no. 3, pp. 159, 2017.

116. L. Razzari, et al., "CMOS-compatible integrated optical hyper-parametric oscillator," Nature Photonics, vol. 4, no. 1, pp. 41-45, 2010.

117. M. Ferrera, et al., "Low-power continuous-wave nonlinear optics in doped silica glass integrated waveguide structures," Nature Photonics, vol. 2, no. 12, pp. 737-740, 2008.

118. M.Ferrera et al.“On-Chip ultra-fast 1 st and 2 nd order CMOS compatible all-optical integration”, Opt. Express, vol. 19, (23)pp. 23153-23161 (2011).

119. D. Duchesne, M. Peccianti, M. R. E. Lamont, et al., "Supercontinuum generation in a high index doped silica glass spiral waveguide," Optics Express, vol. 18, no, 2, pp. 923-930, 2010.

120. H Bao, L Olivieri, M Rowley, ST Chu, BE Little, R Morandotti, DJ Moss, ... Turing patterns in a fiber laser with a nested microresonator: Robust and controllable microcomb generation",Physical Review Research 2 (2), 023395 (2020).

121. M. Ferrera, et al., "On-chip CMOS-compatible all-optical integrator", Nature Communications, vol. 1, Article 29, 2010. 
122. A. Pasquazi, et al., "All-optical wavelength conversion in an integrated ring resonator," Optics Express, vol. 18, no. 4, pp. 3858-3863, 2010.

123. A. Pasquazi, Y. Park, J. Azana, et al., "Efficient wavelength conversion and net parametric gain via Four Wave Mixing in a high index doped silica waveguide," Optics Express, vol. 18, no. 8, pp. 76347641, 2010.

124. M. Peccianti, M. Ferrera, L. Razzari, et al., "Subpicosecond optical pulse compression via an integrated nonlinear chirper," Optics Express, vol. 18, no. 8, pp. 7625-7633, 2010.

125. Little, B. E. et al., "Very high-order microring resonator filters for WDM applications", IEEE Photonics Technol. Lett. 16, 2263-2265 (2004).

126. M. Ferrera et al., "Low Power CW Parametric Mixing in a Low Dispersion High Index Doped Silica Glass Micro-Ring Resonator with Q-factor > 1 Million”, Optics Express, vol.17, no. 16, pp. 1409814103 (2009).

127. M. Peccianti, et al., "Demonstration of an ultrafast nonlinear microcavity modelocked laser", Nature Communications, vol. 3, pp. 765, 2012.

128. A. Pasquazi, et al., "Self-locked optical parametric oscillation in a CMOS compatible microring resonator: a route to robust optical frequency comb generation on a chip," Optics Express, vol. 21, no. 11, pp. 13333-13341, 2013.

129. A. Pasquazi, et al., "Stable, dual mode, high repetition rate mode-locked laser based on a microring resonator," Optics Express, vol. 20, no. 24, pp. 27355-27362, 2012.

130. Wu, J. et al. RF Photonics: An Optical Microcombs' Perspective. IEEE Journal of Selected Topics in Quantum Electronics Vol. 24, 6101020, 1-20 (2018).

131. Xu, X., et al., Photonic microwave true time delays for phased array antennas using a $49 \mathrm{GHz} F S R$ integrated micro-comb source, Photonics Research, 6, B30-B36 (2018).

132. T. G. Nguyen et al., "Integrated frequency comb source-based Hilbert transformer for wideband microwave photonic phase analysis," Opt. Express, vol. 23, no. 17, pp. 22087-22097, Aug. 2015.

133. X. Xu, J. Wu, M. Shoeiby, T. G. Nguyen, S. T. Chu, B. E. Little, R. Morandotti, A. Mitchell, and D. J. Moss, "Reconfigurable broadband microwave photonic intensity differentiator based on an integrated optical frequency comb source," APL Photonics, vol. 2, no. 9, 096104, Sep. 2017.

134. X. Xu, M. Tan, J. Wu, R. Morandotti, A. Mitchell, and D. J. Moss, “Microcomb-based photonic RF signal processing", IEEE Photonics Technology Letters, vol. 31 no. 23 1854-1857, 2019.

135. X. Xu, et al., "Broadband RF channelizer based on an integrated optical frequency Kerr comb source," Journal of Lightwave Technology, vol. 36, no. 19, pp. 4519-4526, 2018.

136. X. Xu, et al., "Continuously tunable orthogonally polarized RF optical single sideband generator based on micro-ring resonators," Journal of Optics, vol. 20, no. 11, 115701. 2018.

137. X. Xu, et al., "Orthogonally polarized RF optical single sideband generation and dual-channel equalization based on an integrated microring resonator," Journal of Lightwave Technology, vol. 36, no. 20 , pp. $4808-4818.2018$. 
138. M.Tan, X. Xu, J. Wu, B. Corcoran, A. Boes, T. G. Nguyen, Sai T. Chu, B. E. Little, R.Morandotti, A. Mitchell, and D. J. Moss, "Integral order photonic RF signal processors based on a soliton crystal micro-comb source", IOP Journal of Optics vol. 23 (11) 125701 (2021).

139. X. Xu, et al., "Advanced adaptive photonic RF filters with 80 taps based on an integrated optical micro-comb source," Journal of Lightwave Technology, vol. 37, no. 4, pp. 1288-1295, 2019.

140. X. Xu, et al., Broadband microwave frequency conversion based on an integrated optical micro-comb source", Journal of Lightwave Technology, vol. 38 no. 2, pp. 332-338, 2020.

141. M. Tan, et al., "Photonic RF and microwave filters based on $49 \mathrm{GHz}$ and $200 \mathrm{GHz}$ Kerr microcombs", Optics Comm. vol. 465,125563, Feb. 22. 2020.

142. X. Xu, et al., "Broadband photonic RF channelizer with 90 channels based on a soliton crystal microcomb", Journal of Lightwave Technology, Vol. 38, no. 18, pp. 5116 - 5121, 2020. doi: 10.1109/JLT.2020.2997699.

143. X. Xu, et al., "Photonic RF and microwave integrator with soliton crystal microcombs", IEEE Transactions on Circuits and Systems II: Express Briefs, vol. 67, no. 12, pp. 3582-3586, 2020. DOI:10.1109/TCSII.2020.2995682.

144. X. Xu, et al., "Photonic RF phase-encoded signal generation with a microcomb source”, J. Lightwave Technology, vol. 38, no. 7, 1722-1727, 2020.

145. X. Xu, et al., "High performance RF filters via bandwidth scaling with Kerr micro-combs," APL Photonics, vol. 4, no. 2, pp. 026102. 2019.

146. M. Tan, et al., "Microwave and RF photonic fractional Hilbert transformer based on a $50 \mathrm{GHz}$ Kerr micro-comb", Journal of Lightwave Technology, vol. 37, no. 24, pp. 6097-6104, 2019.

147. M. Tan, et al., "RF and microwave fractional differentiator based on photonics", IEEE Transactions on Circuits and Systems: Express Briefs, vol. 67, no.11, pp. 2767-2771, 2020.

DOI:10.1109/TCSII.2020.2965158.

148. M. Tan, et al., "Photonic RF arbitrary waveform generator based on a soliton crystal micro-comb source", Journal of Lightwave Technology, vol. 38, no. 22, pp. 6221-6226, Oct 22. 2020. DOI: 10.1109/JLT.2020.3009655.

149. M. Tan, X. Xu, J. Wu, R. Morandotti, A. Mitchell, and D. J. Moss, "RF and microwave high bandwidth signal processing based on Kerr Micro-combs", Advances in Physics X, VOL. 6, NO. 1, 1838946 (2021). DOI:10.1080/23746149.2020.1838946.

150. X. Xu, et al., "Advanced RF and microwave functions based on an integrated optical frequency comb source," Opt. Express, vol. 26 (3) 25692018.

151. Kues, M. et al. Quantum optical microcombs. Nature Photonics 13, (3) 170-179 (2019). doi:10.1038/s41566-019-0363-0

152. P.Roztocki et al., "Complex quantum state generation and coherent control based on integrated frequency combs", Journal of Lightwave Technology 37 (2) 338-347 (2019). 
153. S. Sciara et al., "Generation and Processing of Complex Photon States with Quantum Frequency Combs", IEEE Photonics Technology Letters 31 (23) 1862-1865 (2019). DOI:

10.1109/LPT.2019.2944564.

154. L. Caspani, C. Reimer, M. Kues, et al., "Multifrequency sources of quantum correlated photon pairs on-chip: a path toward integrated Quantum Frequency Combs," Nanophotonics, vol. 5, no. 2, pp. 351-362, 2016.

155. C. Reimer et al., "Generation of multiphoton entangled quantum states by means of integrated frequency combs," Science, vol. 351, no. 6278, pp. 1176-1180, 2016.

156. M. Kues, et al., "On-chip generation of high-dimensional entangled quantum states and their coherent control", Nature, vol. 546, no. 7660, pp. 622-626, 2017.

157. P. Roztocki et al., "Practical system for the generation of pulsed quantum frequency combs," Optics Express, vol. 25, no.16, 18940-18949, 2017.

158. Y. Zhang, et al., "Induced photon correlations through superposition of two four-wave mixing processes in integrated cavities", Laser and Photonics Reviews, vol. 14, no. 7, pp. 2000128, 2020. DOI: 10.1002/Ipor.202000128

159. C. Reimer, et al.,"High-dimensional one-way quantum processing implemented on d-level cluster states", Nature Physics, vol. 15 (2) 148 (2019).

160. H. Bao, et al., Laser cavity-soliton microcombs, Nature Photonics, vol. 13, no. 6, pp. 384-389, Jun. 2019.

161. Bao, C., et al., Direct soliton generation in microresonators, Opt. Lett, 42, 2519 (2017).

162. M.Ferrera et al., "CMOS compatible integrated all-optical RF spectrum analyzer", Optics Express, vol. 22, (18) 21488 (2014).

163. A. Pasquazi, et al., "Sub-picosecond phase-sensitive optical pulse characterization on a chip", Nature Photonics, vol. 5, no. 10, pp. 618-623 (2011).

164. M. Kues, et al., "Passively modelocked laser with an ultra-narrow spectral width”, Nature Photonics, vol. 11, no. 3, pp. 159, 2017.

165. L. Razzari, et al., "CMOS-compatible integrated optical hyper-parametric oscillator," Nature Photonics, vol. 4, no. 1, 41-45, 2010.

166. M. Ferrera, et al., "Low-power continuous-wave nonlinear optics in doped silica glass integrated waveguide structures," Nature Photonics, vol. 2, no. 12, pp. 737-740, 2008.

167. M.Ferrera et al.“On-Chip ultra-fast 1 st and 2 nd order CMOS compatible all-optical integration”, Opt. Express, vol. 19, (23)pp. 23153-23161 (2011).

168. D. Duchesne, M. Peccianti, M. R. E. Lamont, et al., "Supercontinuum generation in a high index doped silica glass spiral waveguide," Optics Express, vol. 18, no, 2, pp. 923-930, 2010.

169. H Bao, L Olivieri, M Rowley, ST Chu, BE Little, R Morandotti, DJ Moss, ... Turing patterns in a fiber laser with a nested microresonator: Robust and controllable microcomb generation",Physical Review Research 2 (2), 023395 (2020). 
170. M. Ferrera, et al., "On-chip CMOS-compatible all-optical integrator", Nature Communications, vol. 1, Article 29, 2010.

171. A. Pasquazi, et al., "All-optical wavelength conversion in an integrated ring resonator," Optics Express, vol. 18 (4) 3858 (2010).

172. A. Pasquazi, Y. Park, J. Azana, et al., "Efficient wavelength conversion and net parametric gain via Four Wave Mixing in a high index doped silica waveguide," Optics Express, vol. 18, no. 8, pp. 76347641, 2010.

173. M. Peccianti, M. Ferrera, L. Razzari, et al., "Subpicosecond optical pulse compression via an integrated nonlinear chirper," Optics Express, vol. 18, no. 8, pp. 7625-7633, 2010.

174. Little, B. E. et al., "Very high-order microring resonator filters for WDM applications", IEEE Phot. Technol. Lett. 16, 2263(2004).

175. M. Ferrera et al., "Low Power CW Parametric Mixing in a Low Dispersion High Index Doped Silica Glass Micro-Ring Resonator with Q-factor > 1 Million", Optics Express, vol.17, no. 16, pp. 1409814103 (2009).

176. M. Peccianti, et al., "Demonstration of an ultrafast nonlinear microcavity modelocked laser", Nature Comm., vol. 3, 765, 2012.

177. A. Pasquazi, et al., "Self-locked optical parametric oscillation in a CMOS compatible microring resonator: a route to robust optical frequency comb generation on a chip," Optics Express, vol. 21, no. 11, pp. 13333-13341, 2013.

178. A. Pasquazi, et al., "Stable, dual mode, high repetition rate mode-locked laser based on a microring resonator," Optics Express, vol. 20, no. 24, pp. 27355-27362, 2012.

179. Xu, X., et al., Photonic microwave true time delays for phased array antennas using a $49 \mathrm{GHz} F S R$ integrated micro-comb source, Photonics Research, 6, B30-B36 (2018).

180. X. Xu, M. Tan, J. Wu, R. Morandotti, A. Mitchell, and D. J. Moss, “Microcomb-based photonic RF signal processing", IEEE Photonics Technology Letters, vol. 31 no. 23 1854-1857, 2019.

181. M. Tan et al, "Orthogonally polarized Photonic Radio Frequency single sideband generation with integrated micro-ring resonators", IOP Journal of Semiconductors, Vol. 42 (4), 041305 (2021). DOI: 10.1088/1674-4926/42/4/041305.

182. Xu, et al., "Advanced adaptive photonic RF filters with 80 taps based on an integrated optical microcomb source," Journal of Lightwave Technology, vol. 37, no. 4, pp. 1288-1295 (2019).

183. X. Xu, et al., Broadband microwave frequency conversion based on an integrated optical micro-comb source", Journal of Lightwave Technology, vol. 38 no. 2, pp. 332-338, 2020.

184. M. Tan, et al., "Photonic RF and microwave filters based on $49 \mathrm{GHz}$ and $200 \mathrm{GHz}$ Kerr microcombs", Optics Comm. vol. 465,125563, Feb. 22. 2020.

185. X. Xu, et al., "Broadband photonic RF channelizer with 90 channels based on a soliton crystal microcomb", Journal of Lightwave Technology, Vol. 38, no. 18, pp. 5116 - 5121, 2020. doi: 10.1109/JLT.2020.2997699. 
186. X. Xu, et al., "Photonic RF and microwave integrator with soliton crystal microcombs", IEEE Transactions on Circuits and Systems II: Express Briefs, vol. 67, no. 12, pp. 3582-3586, 2020. DOI:10.1109/TCSII.2020.2995682.

187. X. Xu, et al., "High performance RF filters via bandwidth scaling with Kerr micro-combs," APL Photonics, vol. 4 (2) 026102. 2019.

188. M. Tan, et al., "Microwave and RF photonic fractional Hilbert transformer based on a $50 \mathrm{GHz}$ Kerr micro-comb", Journal of Lightwave Technology, vol. 37, no. 24, pp. 6097-6104, 2019.

189. M. Tan, et al., "RF and microwave fractional differentiator based on photonics", IEEE Transactions on Circuits and Systems: Express Briefs, vol. 67, no.11, pp. 2767-2771, 2020.

DOI:10.1109/TCSII.2020.2965158.

190. M. Tan, et al., "Photonic RF arbitrary waveform generator based on a soliton crystal micro-comb source", Journal of Lightwave Technology, vol. 38, no. 22, pp. 6221-6226 (2020). DOI: 10.1109/JLT.2020.3009655.

191. M. Tan, X. Xu, J. Wu, R. Morandotti, A. Mitchell, and D. J. Moss, "RF and microwave high bandwidth signal processing based on Kerr Micro-combs", Advances in Physics X, VOL. 6, NO. 1, 1838946 (2021). DOI:10.1080/23746149.2020.1838946.

192. X. Xu, et al., "Advanced RF and microwave functions based on an integrated optical frequency comb source," Opt. Express, vol. 26 (3) 2569 (2018).

193. M. Tan, X. Xu, J. Wu, B. Corcoran, A. Boes, T. G. Nguyen, S. T. Chu, B. E. Little, R.Morandotti, A. Lowery, A. Mitchell, and D. J. Moss, "'Highly Versatile Broadband RF Photonic Fractional Hilbert Transformer Based on a Kerr Soliton Crystal Microcomb", Journal of Lightwave Technology vol. 39 (24) 75817587 (2021).

194. Bao, C., et al., Direct soliton generation in microresonators, Opt. Lett, 42, 2519 (2017).

195. Yuning Zhang, Yang Qu, Jiayang Wu, Linnan Jia, Yunyi Yang, Xingyuan Xu, Baohua Jia, and David J. Moss, "Enhanced Kerr nonlinearity and nonlinear figure of merit in silicon nanowires integrated with 2D graphene oxide films", ACS Applied Materials and Interfaces, Vol. 12 (29) 33094-33103 (2020). DOI:10.1021/acsami.0c07852

196. D.Moss, "11 Tera-FLOP/s photonic convolutional accelerator and deep learning optical neural networks", Research Square, (2021). DOI: https://doi.org/10.21203/rs.3.rs-493347/v1.

197. Moss, David (2020): "11.0 Tera-FLOP/second photonic convolutional accelerator for deep learning optical neural networks", TechRxiv. Preprint. (2020). https://doi.org/10.36227/techrxiv.13238423.v1

198. Xu, X.; Tan, M.; Corcoran, B.; Wu, J.; Boes, A.; Nguyen, T.; Chu, S.; Little, B.; Hicks, D.; Morandotti, R.; Mitchell, A.; Moss, D. "11 Tera-FLOP per Second Photonic Convolutional Accelerator for Deep Learning Optical Neural Networks", Preprints 2020, 2020110420.

199. Moss, David (2020): "RF and microwave photonic high bandwidth signal processing based on Kerr micro-comb sources", TechRxiv. (2020). Preprint. DOI:10.36227/techrxiv.12665609.v3

200. Yuning Zhang, Jiayang Wu, Yunyi Yang, Yang Qu, Linnan Jia, Tania Moein, Baohua Jia, David J. Moss, "Enhanced nonlinear optical figure-of-merit at 1550nm for silicon nanowires integrated with 
graphene oxide layered films", Arxiv (2020). arXiv:2004.08043 [physics.optics]

201. Moss, David; Jia, Baohua; Wu, Jiayang; Zhang, Yuning; Yang, Yunyi; Jia, Linnan, Yang Qu, Tania Moein (2020): "Transforming silicon into a high performing integrated nonlinear photonics platform by integration with 2D graphene oxide films", TechRxiv. (2020). Preprint. DOI:10.36227/techrxiv.12061809.v1.

202. A. Frigg, A. Boes, G. Ren, T.G. Nguyen, D.Y. Choi, S. Gees, D. Moss, A Mitchell, “Optical frequency comb generation with low temperature reactive sputtered silicon nitride waveguides", APL Photonics, Vol. 5 (1), 011302 (2020).

203. M. Tan, X. Xu, J. Wu, A. Boes, B. Corcoran, T. G. Nguyen, S. T. Chu, B. E. Little, R. Morandotti, A. Mitchell, and D. J. Moss, "Advanced applications of Kerr mircocombs", Paper 11775-1. SPIE 11775, Integrated Optics: Design, Devices, Systems and Applications VI, (EOO21) 00107-8, Proc 1177504 (18 April 2021); Integrated Optics Conference, SPIE Optics and Optoelectronics Symposium, Prague, Czech Republic. April 19 - 22 (2021), doi.org/10.1117/12.2588733.

204. T. Moein, D. Gailevičius, T. Katkus, S.H. Ng, S. Lundgaard, D.J. Moss, H. Kurt, Vygantas Mizeikis, K囚stutis Staliūnas, Mangirdas Malinauskas, Saulius Juodkazis, "Optically-thin broadband graphenemembrane photodetector", Nanomaterials, Vol. 10 (3), 407 (2020).

205. Moss, David, "Microcombs for Ultrahigh Bandwidth Optical Data Transmission and Neural Networks." OSF Preprints. March 8. (2021). DOI:10.31219/osf.io/ne9wx.

206. M. Tan, X. Xu, D. J. Moss, "Tera-OPs Photonic Convolutional Neural Networks based on Kerr Microcombs," Preprints, 2021. DOI: 10.20944/preprints202102.0549.v1.

207. M. Tan, X. Xu, D.J. Moss, "Advanced applications of microcombs: From optical neural networks to data transmission," Research Square, April 2021. DOI: 10.21203/rs.3.rs-409803/v1.

208. D. J. Moss et al.,"Tunable dispersion and dispersion slope compensators for $10 \mathrm{~Gb} / \mathrm{s}$ using all-pass multicavity etalons", IEEE Phot. Technology Letters, vol. 15, no. 5, 730-732 (2003).

209. L.M. Lunardi et al." 'Tunable dispersion compensators based on multi-cavity all-pass etalons for 40Gb/s systems", J. Lightwave Technology, vol. 20, (12) 2136 (2002).

210. A. Della Torre et al., "Mid-infrared supercontinuum generation in a low-loss germanium-on-silicon waveguide", APL Photonics Vol. 6, 016102 (2021); doi: 10.1063/5.0033070.

211. M. Sinobad, et al., "Mid-infrared supercontinuum generation in silicon-germanium all-normal dispersion waveguides", Optics Letters, Vol. 45 (18), 5008-5011 (2020). DOI: 10.1364/OL.402159.

212. M. Sinobad et al., "High coherence at $f$ and $2 f$ of a mid-infrared supercontinuum in a silicon germanium waveguide", IEEE Journal of Selected Topics in Quantum Electronics Vol. 26 (2) 8201008 (2020). DOI:10.1109/JSTQE.2019.2943358.

213. M. Sinobad et al., "Dispersion trimming for mid-infrared supercontinuum generation in a hybrid chalcogenide Si-Ge waveguide", Journal of the Optical Society of America B, Vol. 36 (2) A98-A104 (2019). DOI: 10.1364/JOSAB.36.000A98.

214. M. Sinobad et al., "High brightness mid-infrared octave spanning supercontinuum generation to 8.5 $\mathrm{mm}$ in chip-based Si-Ge waveguides", Optica, Vol. 5 (4) 360-366 (2018). 
DOI:10.1364/OPTICA.5.000360.

215. L. Jin et al., Applied Physics Letters Photonics, Vol, 5 Article 056106, (2020). DOI:10.1063/5.0002941

216. L. Carletti et al., "Nonlinear optical properties of Si-Ge waveguides in the mid-infrared", Optics Express Vol. 23 (7) 8261-8271 (2015).

\section{Figures}

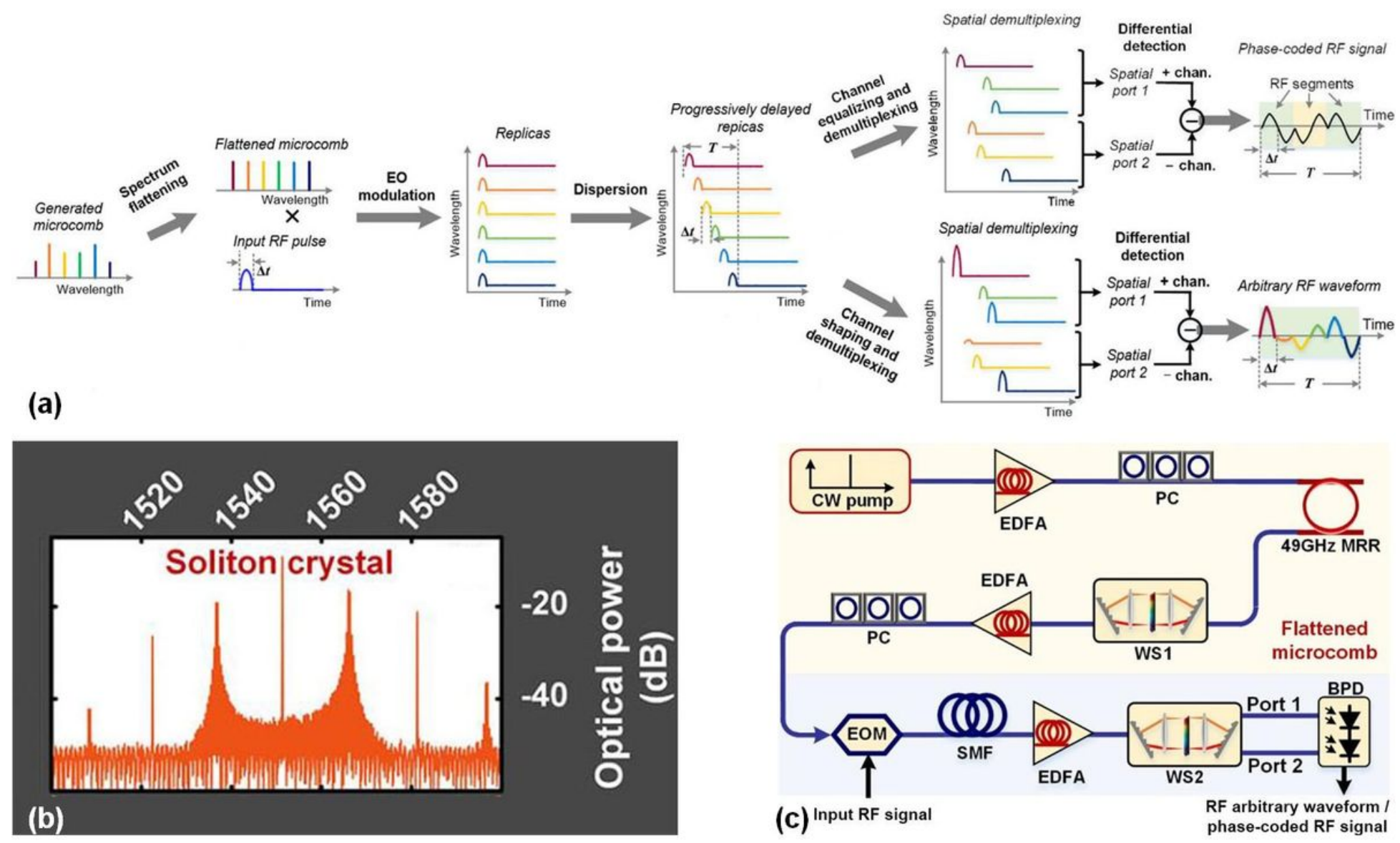

Figure 1

(a) Illustration of the operation of photonic RF phase-encoded signal generator and RF arbitrary waveform generator (AWG). (b) Optical spectrum of soliton crystal micro-comb. (c) Schematic of photonic RF phase-encoded signal generation and arbitrary waveform generation based on an integrated optical micro-comb source. EDFA: erbium-doped fibre amplifier. PC: polarization controller. MRR: microring resonator. WS: WaveShaper. EOM: Mach-Zehnder modulator. SMF: single mode fibre. BPD: balanced photodetector.

Figure 2 
(a),(c),(e) The optical spectra of the flattened micro-comb at different ports of the WaveShaper with different phase codes and sequence length. (b),(d),(f) The assembled phase-encoded RF waveform.
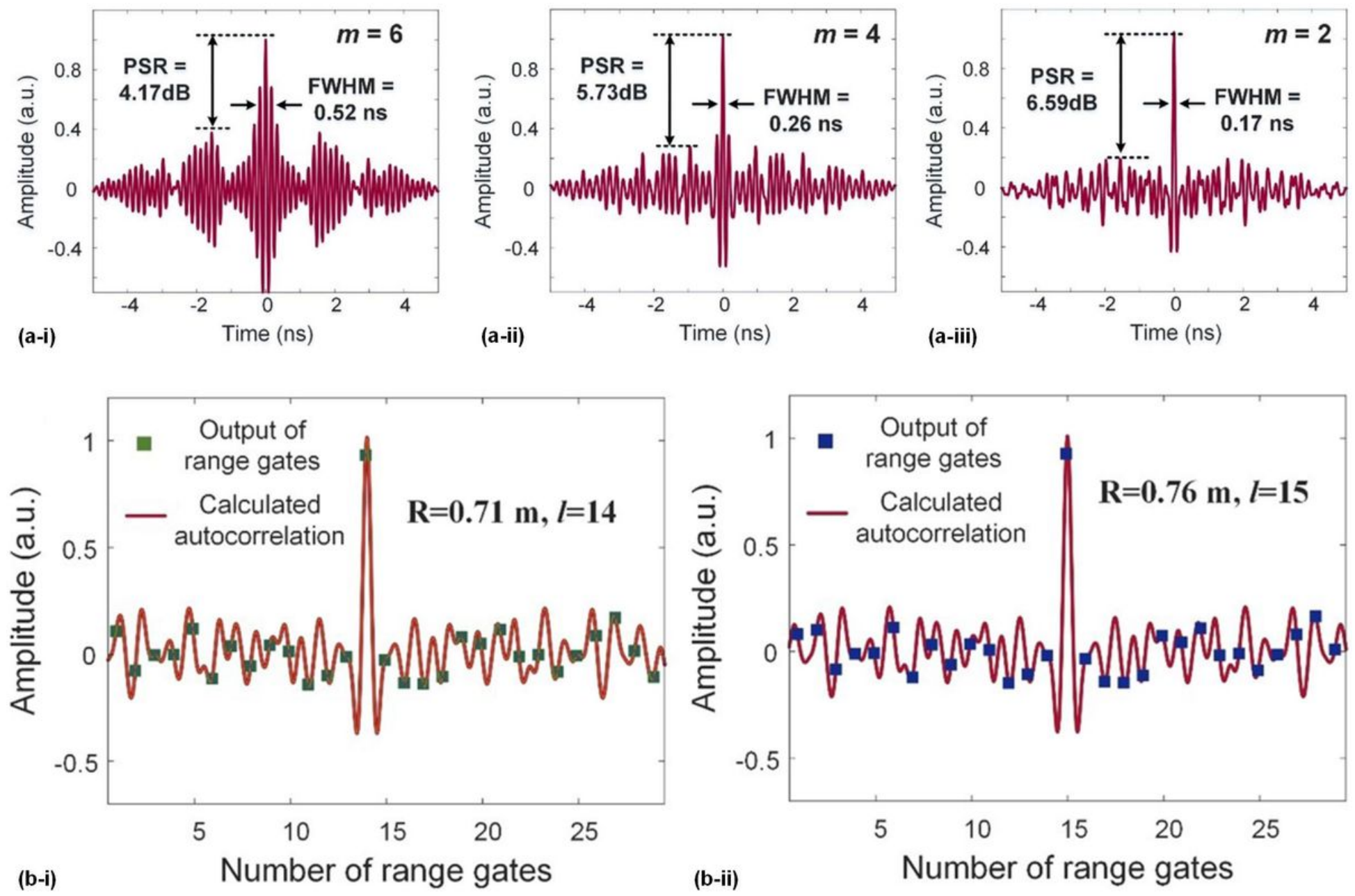

Figure 3

(a) The calculated autocorrelation of the phase-encoded RF waveforms for the number of pulses in each

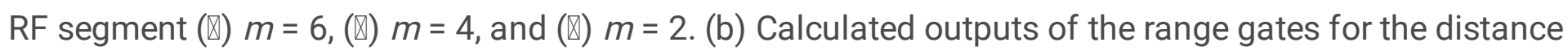
between the target and radar ( ()$R=0.71 \mathrm{~m}$, and (b) $R=0.76 \mathrm{~m}$. 


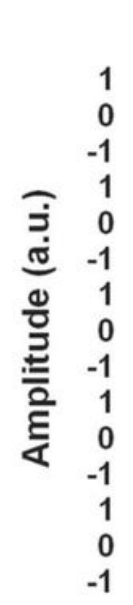

(a-i)

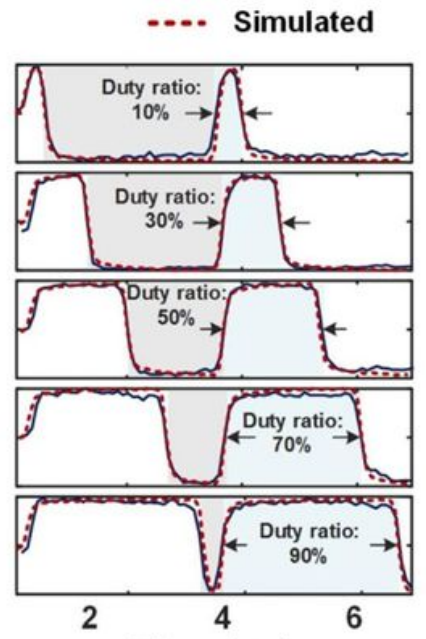

Time (ns)

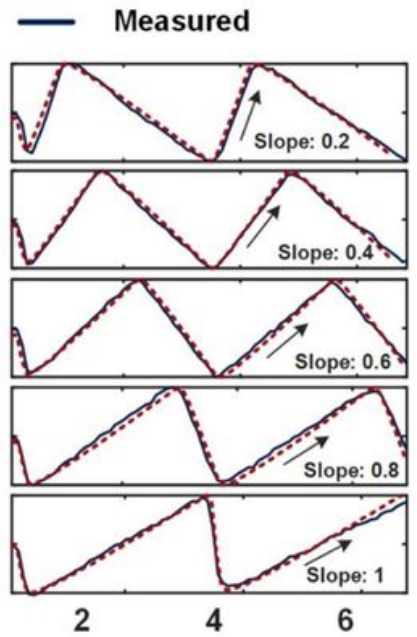

(a-ii) Time (ns)
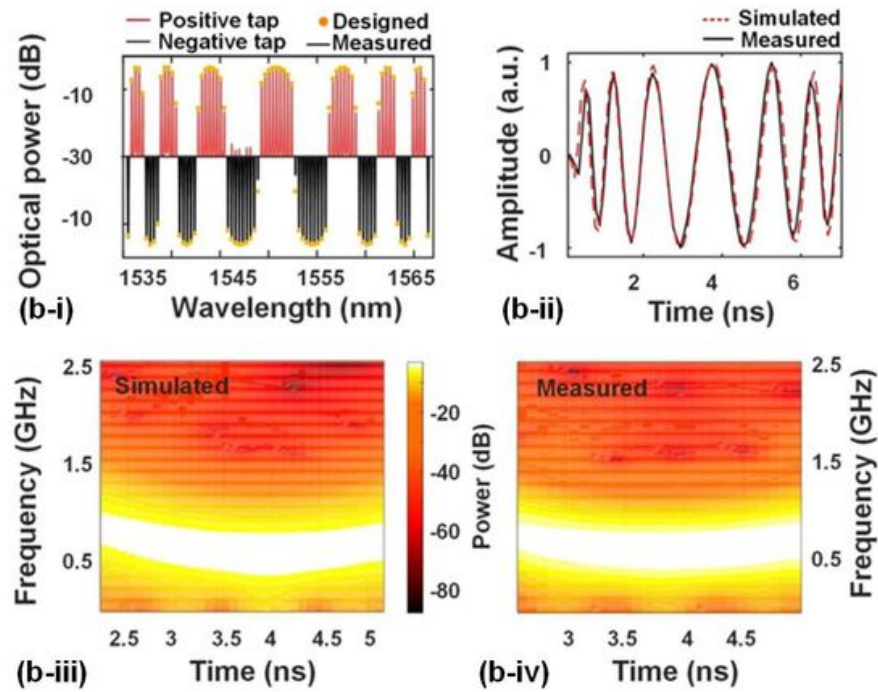

Figure 4

(a) Simulated and measured tunable RF waveforms. ( $($ ) Square waveforms. ( $($ ) Sawtooth waveforms. (b) Experimental results of the generated chirped waveform. ( $(\mathbb{)})$ Designed and measured optical spectra. ( $(\mathbb{)})$ Simulated and measured RF waveforms. The extracted corresponding instantaneous frequency $(\mathbb{})$ simulated and (区) experimental results. 